\title{
Diagnosis of Median Arcuate Ligament Syndrome on Multidetector Computed Tomography
}

\author{
Cemil Göyaa , Cihad Hamidia, b, Salih Hattapoğlua, Mehmet Guli Çetinçakmaka, \\ Memik Teke ${ }^{a}$, Suzan Kuday ${ }^{a}$
}

\begin{abstract}
The median arcuate ligament syndrome (MALS), also known as celiac artery compression syndrome, is a condition which develops secondary to an ischemia in gastrointestinal organs resulting from the compression of proximal celiac truncus by the median arcuate ligament of the diaphragm. It is characterized by postprandial abdominal pain or weight loss. The MALS is conventionally diagnosed by angiography; however, currently the condition can also be diagnosed with multidetector computed tomography (MDCT). In this paper, we aimed to present three cases that were diagnosed with median arcuate ligament syndrome on MDCT.
\end{abstract}

Keywords: Celiac artery; Median arcuate ligament; Multidetector computed tomography

\section{Introduction}

The median arcuate ligament syndrome (MALS) is also known as celiac artery compression syndrome. The incidence of this syndrome is reported to be between 1.76 - 4\% [1-3]. The syndrome is concerned with the ischemia in gastrointestinal organs which develops secondary to the compression of proximal celiac truncus by the median arcuate ligament (MAL) of the diaphragm [4]. In this syndrome, the compression occurs on the proximal part of the celiac artery (especially during the expiration) and thus leads to an abdominal angina and pain [5]. Knowledge about the MALS plays a key role in determining the aetiology of the pain es-

Manuscript accepted for publication July 5, 2013

\footnotetext{
${ }^{a}$ Department of Radiology, Medical School, Dicle University, Diyarbakir, Turkey

${ }^{\mathrm{b} C}$ Corresponding author: Cihad Hamidi, Department of Radiology, Medical School, Dicle University, Yenisehir, 21280, Diyarbakir, Turkey.Email: cihad5@hotmail.com
}

doi: http://dx.doi.org/10.4021/jmc1384w pecially in the cases of chronic abdominal pain, for which no aetiological reason can be established.

In this paper, we aimed to present three cases that were diagnosed with median arcuate ligament syndrome on multidetector computed tomography (MDCT).

\section{Case Report}

\section{Case 1}

A 39-year-old woman presented to our general surgery department once she had been diagnosed with a hepatic mass on ultrasound scanning in another hospital. A three-phase abdominal computed tomography (CT) was obtained in order to characterize the hepatic mass. Using a 64-slice scanner (Philips Brilliance CT scanner; Philips Medical Systems, Cleveland, OH, USA), a volume of $120 \mathrm{~mL}(300 \mathrm{mg} / \mathrm{mL})$ of non-ionic contrast medium was injected at a speed of 5.0 $\mathrm{mL} / \mathrm{s}$, and then slices were obtained beginning from the diaphragm level to the bifurcation of the iliac arteries. Angiography was performed with the following scan parameters: slice increment: $0.90 \mathrm{~mm}$; pitch 0.9 , gantry rotation time: 0.5 seconds, and delay time: 18 - 20 seconds. The images were then transferred to the work station. The hepatic masses detected on ultrasonography (US) were regarded as haemangioma. Besides these, reformatted axial and sagittal CT images were obtained for the slices taken from the arterial phase. The images revealed a dilatation which was resulting from the compression and narrowing of the celiac artery by the MAL. The hooked appearance of the proximal celiac truncus was observed on the sagittal images. Also, the compression of MAL and celiac artery were clearly seen on axial (Fig. 1a) and sagittal (Fig. 1b) MDCT scans.

\section{Case 2}

A 77-year-old man presented with chronic abdominal pain, which had been ongoing for 15 years. The pain was localized in the epigastric region and occurred after meals and nausea. The physical examination was uneventful except for the epigastric tenderness. No abnormality was detected in the pre- 

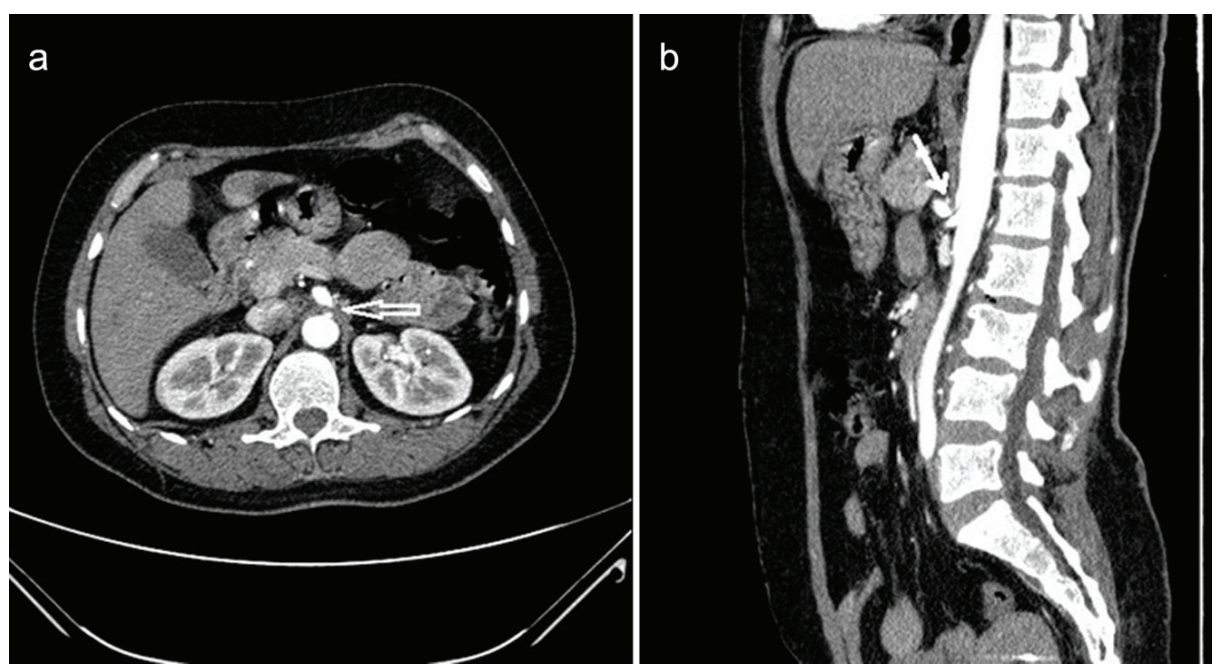

Figure 1. Reformatted axial (a) and sagittal (b) images (in the 39-year-old woman with MAL syndrome) show the compression of the celiac artery by the hypertrophic MAL, a narrowing in the celiac trunk lumen, and a poststenotic dilatation (arrow). The sagittal image shows a typical hooked appearance of the proximal celiac trunk.

viously obtained upper gastrointestinal system endoscopy. A hepatic haemangioma and also a cortical cyst in the right kidney were detected on the abdominal US. Depending on ischemic symptoms, a CT angiography was performed for screening abdominal artery and its branches. The compression of celiac artery by the MAL was clearly visible on the $\mathrm{CT}$ angiography (Fig. 2). Mixed atheromatous plaques were present in the abdominal aorta, beginning from the infrare-

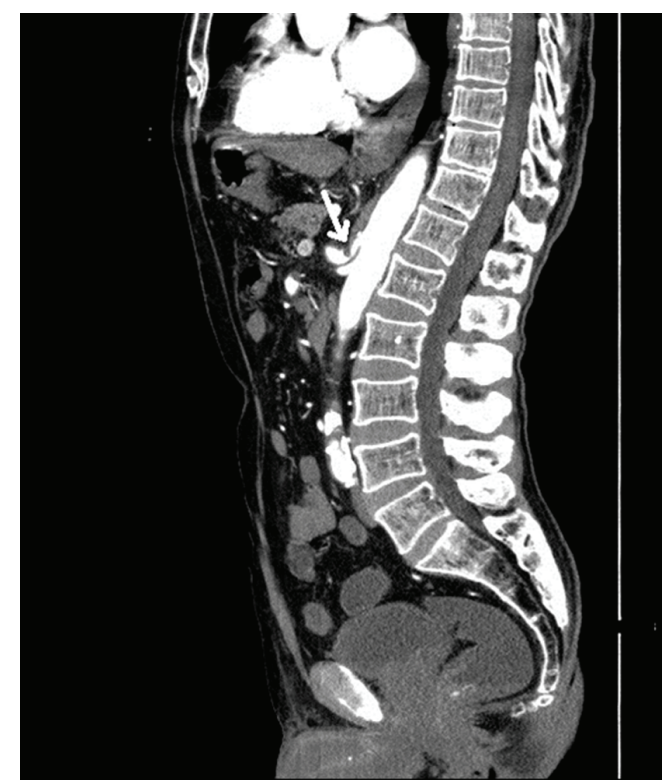

Figure 2. The reformatted sagittal CT image (in the 77-yearold man with MAL syndrome) shows the compression of the celiac artery by the hypertrophic MAL and a narrowing in the celiac trunk lumen (arrow). The sagittal image shows a typical hooked appearance of the proximal celiac artery. nal level. The lumens of the superior and inferior mesenteric arteries were open.

\section{Case 3}

A 73-year-old woman presented with chronic abdominal pain, which had been ongoing for 5 years. The epigastric pain was not associated with eating, and the physical examination was uneventful, except for epigastric tenderness.

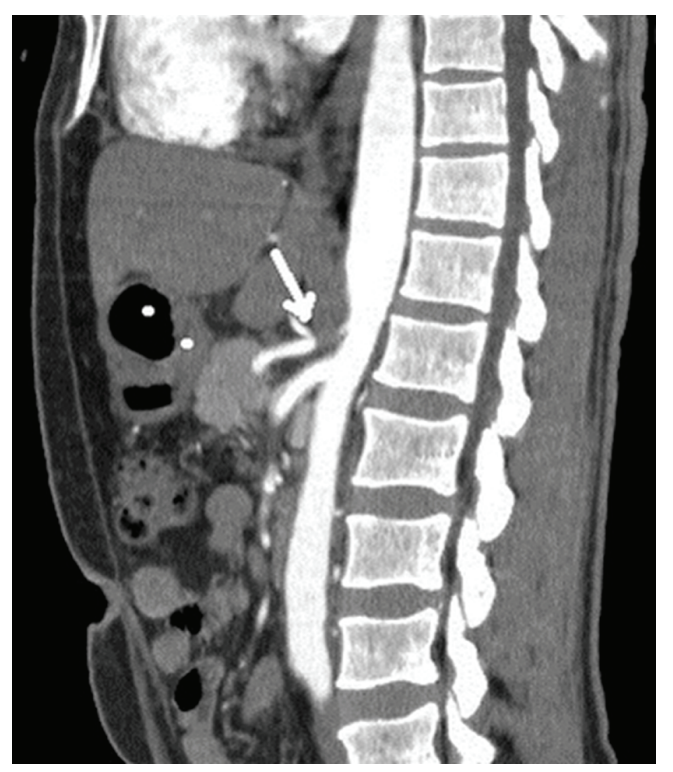

Figure 3. The reformatted sagittal CT image (in the 73-yearold woman with MAL syndrome) shows the compression of the celiac artery by the hypertrophic MAL and a narrowing in the celiac trunk lumen (arrow). 
The abdominal US revealed haemangioma-like hyperechoic lesions in the liver. A three-phase abdominal CT was performed for the mesenteric ischemia and the hepatic mass. On the abdominal CT, the hepatic lesions were confirmed as haemangioma. The lumens of the superior and inferior mesenteric arteries were viewed as open on the reformatted axial and sagittal CT images. The compression of the celiac artery by the hypertrophic MAL and a narrowing in the celiac trunk were also visible (Fig. 3). Depending on these findings, the diagnosis was confirmed as MALS.

\section{Discussion}

The MAL is a fibrous arch which connects the two diaphragmatic crura that are located on both sides of the aorta. This ligament may cause a compression and abdominal pain while passing anterior to the proximal part of the celiac truncus [6]. The MALS was initially described by Harjola, in 1963 [7]. Although the syndrome was described in the 1960s, there is an ongoing debate as to whether celiac compression leads to the clinical picture or not. The controversy arises from an undefined pathophysiological mechanism and the existence of celiac compression in asymptomatic patients [8]. It has been reported that $13 \%-50 \%$ of healthy individuals may exhibit angiographically different features of compression especially during expiration. The MALS is most commonly seen in young and female patients aged between 20 and 40 years. The syndrome is characterized by typical postprandial signs including epigastric pain, weight loss, and nausea. The abdominal pain usually starts within the 15 - 30th minutes after eating. In the cases with MALS, the celiac artery is narrowed by the MAL during expiration [5]. In inspiration, however, the celiac artery descends to the abdominal cavity, and compression is often relieved due to the more vertical orientation of the celiac artery [6]. Celiac, superior, and inferior mesenteric arteries are the fundamental vascular structures that supply the intestinal ansae, and there is a perfect collateral circulation among them. Most of the MALS cases remain asymptomatic due to the collateral circulation supplied from the superior mesenteric artery [9]. There are two main theories used to explain the pathogenesis of the symptoms. The first one is the mesenteric ischemia arising from celiac artery compression. The second theory is the neurogenic stimulation that is caused by the compression of celiac ganglion and plexus $[10,11]$.

Celiac artery compression may be investigated with Doppler US, conventional angiography, CT angiography, and magnetic resonance angiography. Doppler US has been reported to have a high sensitivity for the diagnosis of the MALS and has been proposed to be the method of choice for diagnosing the MALS. The gold standard diagnostic method is conventional angiography, which should be performed during both inspiration and expiration, in the lateral position. The images that are not taken in the lateral position may fail to visualize the lesion and the MAL $[5,10,12]$. However, recent implementation of MDCT has enabled the production of fast, thin-slice, multiplanar, reformatted and 3D angiographic images. Also, high-resolution images can be obtained for the aorta and its branches and also for the mesenteric vascular structures [6]. In our study with 64-slice $\mathrm{CT}$, the multiplanar reformatted 3D angiographic images perfectly demonstrated the vascular anatomy and compression without any need for conventional angiography, which is a more invasive technique. In patients with MAL syndrome, CT angiography demonstrates a characteristic focal narrowing in the proximal celiac axis. The focal narrowing has a characteristic hooked appearance, which can be useful in distinguishing this condition from other causes of celiac artery stenosis such as atherosclerosis. The features of the MALS include poststenotic dilatation and collateral vascular vessels.

The treatment of the MALS remains controversial. Surgical treatment of MAL syndrome is performed in the ages between 40 and 60 in order to eliminate the symptoms such as postprandial abdominal pain, weight loss, poststenotic dilatation, and collateral vascular vessels [6]. The options of treatment for the MALS include surgical or laparoscopic division of the celiac ganglion destruction and ganglion blockage [10].

We conclude that the MDCT is a minimally invasive and useful diagnostic tool for the diagnosis of MAL syndrome, which can be used as an alternative to the conventional invasive angiography.

\section{References}

1. Ilica AT, Kocaoglu M, Bilici A, Ors F, Bukte Y, Senol A, Ucoz T, et al. Median arcuate ligament syndrome: multidetector computed tomography findings. J Comput Assist Tomogr. 2007;31(5):728-731.

2. Park CM, Chung JW, Kim HB, Shin SJ, Park JH. Celiac axis stenosis: incidence and etiologies in asymptomatic individuals. Korean J Radiol. 2001;2(1):8-13.

3. Gumus H, Gumus M, Tekbas G, Onder H, Ekici F, Cetincakmak MG, Bilici A. Clinical and multidetector computed tomography findings of patients with median arcuate ligament syndrome. Clin Imaging. 2012;36(5):522-525.

4. Karahan OI, Kahriman G, Yikilmaz A, Ok E. Celiac artery compression syndrome: diagnosis with multislice CT. Diagn Interv Radiol. 2007;13(2):90-93.

5. Wolfman D, Bluth EI, Sossaman J. Median arcuate ligament syndrome. J Ultrasound Med. 2003;22(12):13771380.

6. Horton KM, Talamini MA, Fishman EK. Median arcuate ligament syndrome: evaluation with $\mathrm{CT}$ angiogra- 
phy. Radiographics. 2005;25(5):1177-1182.

7. Harjola PT. A Rare Obstruction of the Coeliac Artery. Report of a Case. Ann Chir Gynaecol Fenn. 1963;52:547550 .

8. Szilagyi DE, Rian RL, Elliott JP, Smith RF. The celiac artery compression syndrome: does it exist? Surgery. 1972;72(6):849-863.

9. Lee V, Alvarez MD, Bhatt S, Dogra VS. Median arcuate ligament compression of the celiomesenteric trunk. J Clin Imaging Sci. 2011;1:8.
10. Cina CS, Safar H. Successful treatment of recurrent celiac axis compression syndrome. A case report. Panminerva Med. 2002;44(1):69-72.

11. Tribble CG, Harman PK, Mentzer RM. Celiac artery compression syndrome. Report of a case and rewiev of current opinion. Vasc Surg. 1986;20(2):120-129.

12. Kopecky KK, Stine SB, Dalsing MC, Gottlieb K. Median arcuate ligament syndrome with multivessel involvement: diagnosis with spiral CT angiography. Abdom Imaging. 1997;22(3):318-320. 